\title{
Caracterización del comportamiento y las señales vibratorias en Euthyrhynchus floridanus (Hemiptera: Pentatomidae) durante el cortejo y la cópula
}

\author{
R. Daniel Briceño \\ Escuela de Biología, Universidad de Costa Rica, San José, Costa Rica; daniel.briceno@ucr.ac.cr \\ Recibido 25-II-2013. Corregido 10-VII-2013. Aceptado 20-VIII-2013.
}

\begin{abstract}
Characterization of the behavioral and vibrational signals in Euthyrhynchus floridanus (Hemiptera: Pentatomidae) during courtship and copulation. Euthyrhynchus floridanus (Linnaeus) (Hemiptera: Pentatomidae) is a neotropical species belonging to the family Pentatomidae with over 4000 species described, and is distributed from Florida to Brazil. This study describes the sexual behavior and reported for the first time the production of substrate vibrations by males and females during copulatory behavior and mating. Courtship and copulatory behavior, as well as the diverse signals, were recorded with a phonographic cartridge connected to a video camera. Female vibrations were reproduced in the absence of females and the responses by males were recorded. At least three types of substrate vibrations were distinguished in males and one in females, and these signals were characterized by their low frequency, varying from $127 \mathrm{to} 180 \mathrm{~Hz}$. The sounds of E. floridianus males were significantly different in frequency, duration and number of pulses, both in courtship and in copulation, for the purring and drumming sounds. The production of sounds in this species is associated principally with mechanical, stimulatory behavior during courtship and copulation. Patterns of behavior and their relation to substrate vibrations suggest that these signals are important for the males in the context of mate location and sexual selection. Rev. Biol. Trop. 62 (1): 95-107. Epub 2014 March 01.
\end{abstract}

Key words: Euthyrhynchus floridanus, Hemiptera, stink bugs, substrate vibrations, courtship, mating.

Los insectos suelen intercambiar información por medio de varios tipos de señales: feromonas, danzas aéreas, diversos tipos de cantos, entre otros (Stewart 1997). Sin embargo, se ha determinado que la comunicación por vibraciones de sustrato se relacionan con muchos tipos de comportamientos, entre los que se encuentran las señales de cortejo y apareamiento (Cocroft, 2001).

La comunicación a través de vibraciones transmitidas por el substrato se ha estudiado por largo tiempo, pero en comparación con sonidos transmitidos por el aire ha recibido muy poca atención. Sin embargo, en años recientes se ha puesto en claro que estas señales juegan un papel crucial en la comunicación en muchos grupos de insectos relacionándolas con aspectos de la conducta sexual, alarma, defensiva, para coordinar acciones de grupo e interacciones sociales complejas (Rovner, 1975; Kalmring \& Elsner, 1985; Barth, 2002; Vibrant-Doberlet \& Čokl, 2004; Cocroft \& Rodríguez, 2005).

En insectos, las señales transmitidas por sustrato son producidas por métodos diversos, y son detectadas por receptores localizados en las patas (Vibrant-Doberlet \& Cokl, 2004). Las señales son producidas principalmente por mecanismos de estridulación así como por vibraciones de alguna parte del cuerpo, lo que puede conllevar a la producción de muchos tipos de señales, que varían de acuerdo a sus características físicas (Thorpe \& Harrington, 1981). Las velocidades de propagación con que las ondas producidas se transmiten van a depender tanto de la frecuencia como de las 
propiedades mecánicas del sustrato; usualmente, las plantas causan una muy baja atenuación de las señales, lo que permite amplios rangos de comunicación (Cokl \& VibrantDoberlet, 2001)

Dentro del orden Hemiptera se han realizado múltiples grabaciones y análisis de sonidos transmitidos por vibraciones de sustrato para muchas especies de diversas familias, entre ellos la familia Pentatomidae. Sin embargo, son pocos los estudios que relacionan el comportamiento con estos sonidos (Haskell, 1953; Moore, 1961; Thorpe \& Harrington, 1981) por lo que se conoce poco sobre su probable función. La conducta de cortejo del chinche hediondo Nezara viridula (L.) (Hemiptera: Pentatomidae) se ha tomado como modelo representativo para ilustrar algunos mecanismos de comunicación por vibraciones de sustrato en insectos (Virant-Doberlet \& Cokl, 2004).

Euthyrhynchus floridanus (Linnaeus) es una especie neotropical perteneciente a la familia Pentatomidae con mas de 4000 especies descritas, y se distribuye desde la Florida hasta Brasil (Arismendi \& Thomas, 2003; McPherson \& McPherson, 2010). A diferencia de otras subfamilias de Pentatomidae, todos de hábitos fitófagos, los Asopinae son esencialmente depredadores (De Clercq, 2000). Es considerado un insecto beneficioso porque se le reporta como depredador de especies de interés económico como Nezara viridula (Linnaeus), Papilio cresphontes (Cramer), Anticarsia gemmatalis (Hübner), Leptinotarsa decemlineata (Say), Diaprepes sabbreviatus (Linnaeus), entre otras (Meed \& Richman, 2000).

Este estudio describe el comportamiento sexual de E. floridianus y se reporta por primera vez en esta especie la producción de vibraciones de sustrato por machos y hembras durante el comportamiento precopulatorio y la cópula.

\section{MATERIALES Y MÉTODOS}

Cría E. floridianus: El periodo de estudio abarcó de setiembre del 2005 a octubre del 2007. El cultivo de las chinches en el laboratorio se inició con ninfas y adultos recolectados en el Valle Central de Costa Rica (San Antonio de Escazú, Lambert $521300206600 \mathrm{~N}$ ). Machos (de tamaño entre 11.5 a $13.7 \mathrm{~mm})$ y hembras (12 a 17 $\mathrm{mm}$ ) de E. floridianus se criaron en jaulas de madera con tela de malin ( $43 \mathrm{~cm}$ de alto, largo y de ancho) bajo fotoperiodos de $12 \mathrm{~L}: 12 \mathrm{~N} \mathrm{a}$ temperatura y humedad ambiental. Durante el primer estadio, las ninfas no se alimentaron, consumiendo solamente agua; posteriormente las ninfas se alimentaron con moscas Drosophila sp. y Musca domestica, mientras que las ninfas de quinto estadio se alimentaron con cucarachas adultas de Periplaneta australiacea y $P$. americana recolectadas en casas. Una vez que las ninfas emergieron como adultos se separaron individualmente en vasos de plástico que contenían una esponja impregnada de agua. Los animales se alimentaron de dos a tres veces al día con moscas del mediterráneo (Ceratitis capitata). Las hembras y machos maduraron sexualmente después de dos semanas. El ciclo total desde huevo a adulto fue de 60 días aproximadamente.

Observaciones del cortejo y la cópula: Se grabó tanto el comportamiento de cortejo y de cópula como las diversas señales producidas utilizando un cartucho fonográfico Astatic 91T y un amplificador, conectados a una cámara de video Sony DCR-TRV 80. Las grabaciones se realizaron entre las 8 horas y las 12 medio día, periodo en el cual los animales fueron más activos. Una hembra madura virgen se colocó sobre una planta de frijol común (Phaseolus vulgaris) en maceta y se esperó por 15 minutos; inmediatamente después se colocó un macho en la planta, y se ubicó el cartucho fonográfico aproximadamente a $10 \mathrm{~cm}$ de la hembra. Las señales se digitalizaron $(22 \mathrm{Khz})$ y se analizaron con el programa AvisoftSaslab Pro® ${ }^{\circledR}$ 4.15. Se observó el cortejo de 42 parejas de las cuales solamente 20 resultaron en cópulas y fueron las que se utilizaron para el análisis. Los parámetros acústicos medidos para cada canto fueron: frecuencia $(\mathrm{Hz})$, duración promedio de tiempo por elemento, diferencia de tiempo entre 
elementos y número de elementos por canto para cada cópula.

\section{Experimentos con sonidos de las hem-}

bras: Para reproducir las vibraciones de las hembras se modificó un parlante de radio Shack Mini Speaker de $29 \mathrm{~mm}$ y $80 \mathrm{Ohm}$, pegando al centro y en dirección perpendicular a la membrana, un palito de balsa de $2 \times 2 \mathrm{~mm}$ de grosor y $1 \mathrm{~cm}$ de largo. El resto de la membrana (menos una banda angosta que cubre los alambres de cobre que van hacia el centro del parlante, que se sostuvo con una cinta adhesiva eléctrica) se quitó con una navaja de disección, para disminuir al máximo la producción de sonido aéreo. La punta del palito de balsa se colocaba en contacto con el tallo o vena central de una hoja, sosteniendo el parlante con una base metálica que se colocaba junto a la planta. El parlante se conectó a la salida de audio de una computadora con tarjeta de sonido NeoMagicMagic Media 256AV. Con el programa AvisoftSaslab Pro ${ }^{\circledR} 4.15$ se determinó la intensidad promedio (db) de al menos dos segmentos de las señales de llamada de cada hembra. Posteriormente, se reprodujo esta señal en ausencia de la hembra y se registró las respuestas de los machos. Para no producir un estímulo más fuerte del natural, los experimentos se hicieron con el volumen nivel tres donde seis es el máximo. Para el análisis estadístico se utilizaron las pruebas de U Mann Whitney y KolgomorovSmirnov.

\section{RESULTADOS}

Descripción del comportamiento precopulatorio y copulatorio: Los comportamientos y posiciones del macho y la hembra observados durante el cortejo y la copula se describen en detalle en el cuadro 1 . Se documentó un total de 6892 actos en 20 cópulas. Las transiciones más frecuentes entre comportamientos y sonidos se resumen en la Fig. 2 en que los números se refieren a porcentajes de todas las transiciones.

Durante el cortejo en el 95\% $(n=19)$ de los casos los machos se detuvieron y realizaron de una a varias sacudidas rápidas del cuerpo mientras se acercaron a las hembras.
Este movimiento se ejecutó lejos (36\%), cerca $(46 \%)$ o sobre la hembra (18\%). De estos machos, 14 lo hicieron sin ningún contacto físico previo con la hembra y cinco después de que las tocaron con sus antenas.

El macho se acercó a la hembra en una de dos formas: ya sea por el frente $(52 \%, n=10)$ de modo que el macho y la hembra se ubicaron cabeza con cabeza, o desde la parte posterior $(26 \%, n=5)$ donde la cabeza del macho se situó frente a la parte posterior de la hembra, y en los restantes cuatro casos, la hembra se acercó al macho pero sólo desde la parte frontal. Una vez que el macho estableció contacto con la hembra, este inició inmediatamente un vigoroso toqueteo con sus antenas. Un alto porcentaje de hembras cortejadas $(53 \%, \mathrm{~N}=22)$ se negó a copular y huyó durante esta fase en dirección contraria. En cuatro de estas ocasiones, las hembras empujaron a los machos lejos de su parte posterior con su patas metatoráxicas.

Cuando ambos sexos se encontraron de frente, el macho tocó usualmente con sus antenas el primer par de patas o las antenas de la hembra y sacudió el cuerpo rápidamente varias veces a ambos lados (Fig. 1A). La cópula se realizó correctamente cuando la hembra se mantuvo estacionaria después del primer contacto. El macho se montó por un costado del cuerpo de la hembra y una vez orientado con sus antenas, le tocó la cabeza y antenas. Luego, el macho abrió sus alas moviendo el primer par hacia delante mientras frotó con las patas segundas los lados del tórax y el abdomen de la hembra (Fig. 1B); posteriormente, el plegó las antenas hacia atrás. Durante los frotes y toques con sus patas, el macho sacudió rápidamente su cuerpo a ambos lados, se detuvo regularmente y movió rápidamente dorso ventralmente la genitalia parcialmente extendida (Fig. 1C). Posteriormente, el dobló la punta del abdomen bajo el de la hembra, lo que le permite a los genitales hacer contacto e introducir su órgano copulatorio en la apertura genital. La duración promedio de la cópula fue de $97 \pm 45$ $\min (n=20)$.

La secuencia normal de comportamientos $\mathrm{y}$ frecuencia durante la cópula fue muy 


\section{CUADRO 1}

Etograma . Posiciones y unidades de comportamientos observados durante el cortejo y la cópula de machos y hembras en E. floridianus.

TABLE 1

Ethogram. Positions and behaviors units observed during courtship and copulation of males and females in E. floridianus.

\section{CORTEJO}

Macho

Aproximarse: Se aproxima por detrás, de frente, o el lado de la hembra.

Toque antenas: Toca con sus antenas la parte posterior dorsal del cuerpo, las patas, la cabeza o las antenas de la hembra.

Sacude el cuerpo: sacude el cuerpo rápidamente varias veces a ambos lados en posición estacionaria.

Monta: Se montó por detrás o el lado del cuerpo de la hembra.

Estacionario: el macho permanece estacionario y no se mueve.

Abre y cierra alas: Abrió sus alas moviendo el primer par hacia delante, posteriormente pliega las antenas hacia atrás.

Detenerse: deja de caminar.

Extiende genitalia: Extiende parcialmente la genitalia.

Mueve genitalia: Mueve la genitalia extendida rápidamente dorso ventralmente.

Gira cuerpo $180^{\circ}$ : Gira $180^{\circ}$ grados el cuerpo alejando la cabeza de la parte posterior de la hembra orientando ambos puntas de sus abdómenes hacia el otro.

Dobla punta del abdomen: Dobla la punta del abdomen y trata de insertar su genitalia en la apertura genital de la hembra.

Hembra

Estacionaria: Permanece estacionaria sin moverse.

Aproximación: Se aproxima hacia el macho.

Alejarse: Se aleja en dirección opuesta al macho.

Empujar: Empuja al macho lejos de su parte posterior con su patas metatoráxicas.

Vibra hemiélitros: Levantó sus hemiélitros y los separó ligeramente del abdomen haciéndolos vibrar.

Macho

\section{CÓPULA}

Frote patas: Mientras esta en la posición 180 con el pygoporo girado, el macho utiliza las patas metatoráxicas para localizar y frotar regularmente y activamente los lados del abdomen de la hembra.

Vibrar cuerpo: El macho vibra ligeramente el cuerpo a los lados.

Elevar abdomen: El macho eleva su abdomen sobre el eje medio de su cuerpo y permanecen por algunos segundos en esa posición.

Mover élitros: El macho mueve los élitros ligeramente dorso ventralmente.

Desenganchar genitalia: De la posición punta-punta, el macho vuelve a la posición 0 mientras gira el pygoporo a su posición original y desengancho su genitalia. Posteriormente ambos se separaron.

Zumbar alas: Vibración corta de las alas hacia delante.

Hembra

Camina: Durante la copula la hembra camina y el macho la sigue caminado hacia atrás.

Agitar abdomen: Agitar amplia y fuertemente el abdomen a ambos lados.

estereotipada y puede ser observada de las Figs. 2 y 3 . Una vez asumida la posición de cópula el macho frotó activa y regularmente con sus patas posteriores los lados del abdomen de la hembra (Figs. 1D, 2a, 3), alternando con el levantamiento del abdomen de ambos, y permaneciendo por algunos segundos en esa posición (Figs. 1D, 2a, 3). Estas dos actividades se coordinaron y sumadas representaron el $56 \%$ de los comportamientos observados para los machos durante la cópula (Fig. 2a). A partir de esta posición, se observó al macho mover los élitros ligeramente dorso ventralmente asociado con la producción de sonidos (Fig. 1E). 


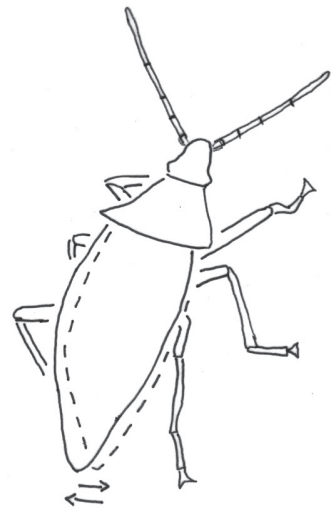

A

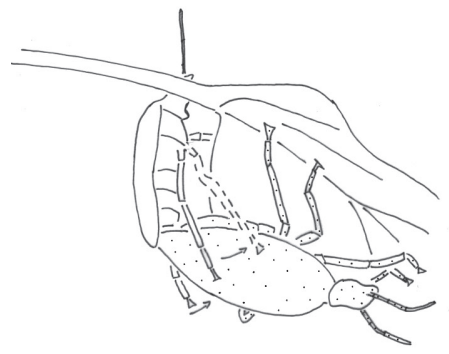

D

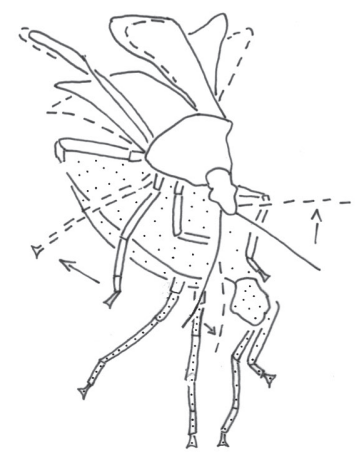

B

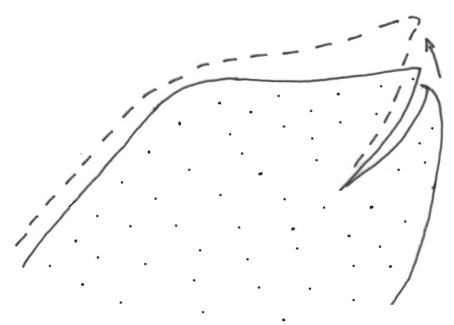

$\mathrm{E}$

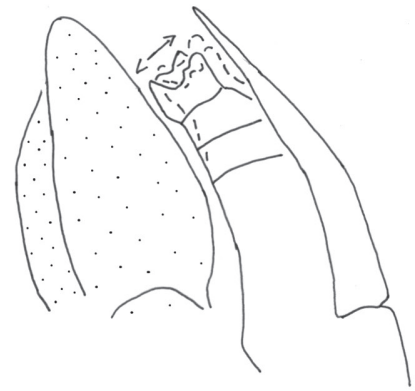

C

Fig. 1. Comportamientos del macho y la hembra (punteada) durante el cortejo y la cópula. Línea discontinua indica posición siguiente.

Fig. 1. Male and female (dotted) behavior during the courtship and mating. Dashed line indicates next position.

Frecuentemente (18\%) el macho zumbo las alas antes o después de frotar el abdomen de la hembra y en un $5 \%$ de las ocasiones fue seguida de un segundo zumbido (Fig. 2a, 3), alternando con vibraciones suaves y cortas del cuerpo (Figs. 2a, 3). Cerca del final de la cópula, la hembra agitó regularmente y fuertemente el abdomen a ambos lados (Fig. 1F); este movimiento se alternó con el frote de las patas, por parte del macho, de los lados del abdomen de la hembra. El macho a continuación desenganchó su genitalia y ambos se separaron.

Señales vibratorias producidas durante el comportamiento precopulatorio y copulatorio: Una serie de pulsos fueron observadas en oscilogramas durante el cortejo y la cópula en E. floridianus. Se distinguieron al menos tres vibraciones de sustrato distintas para los machos y una para las hembras, y se caracterizaron por ser señales de baja frecuencia (Fig. 4 y 5). La secuencia de los sonidos producidos por los machos durante la cópula se observan en la figura $2 \mathrm{~b}$ y se ilustran en la figura 5 . En los machos, fue frecuente que después de producir el sonido del tamborileo hicieran el ronroneo seguido del zumbido con las alas. Todas las señales, a excepción del batido de alas, no fueron sonoras.

Señales producidas por los machos. Sacudidas del cuerpo: Las sacudidas rápidas del cuerpo, presentes solo durante el cortejo, generaron vibraciones con una frecuencia promedio de $136 \pm 130 \mathrm{~Hz}$ y una duración de $0.46 \pm 0.05 \mathrm{~s} \quad(\mathrm{n}=10)$ (Fig. 1a y 2a) y representaron el $41 \%$ del total de los sonidos registrados. 


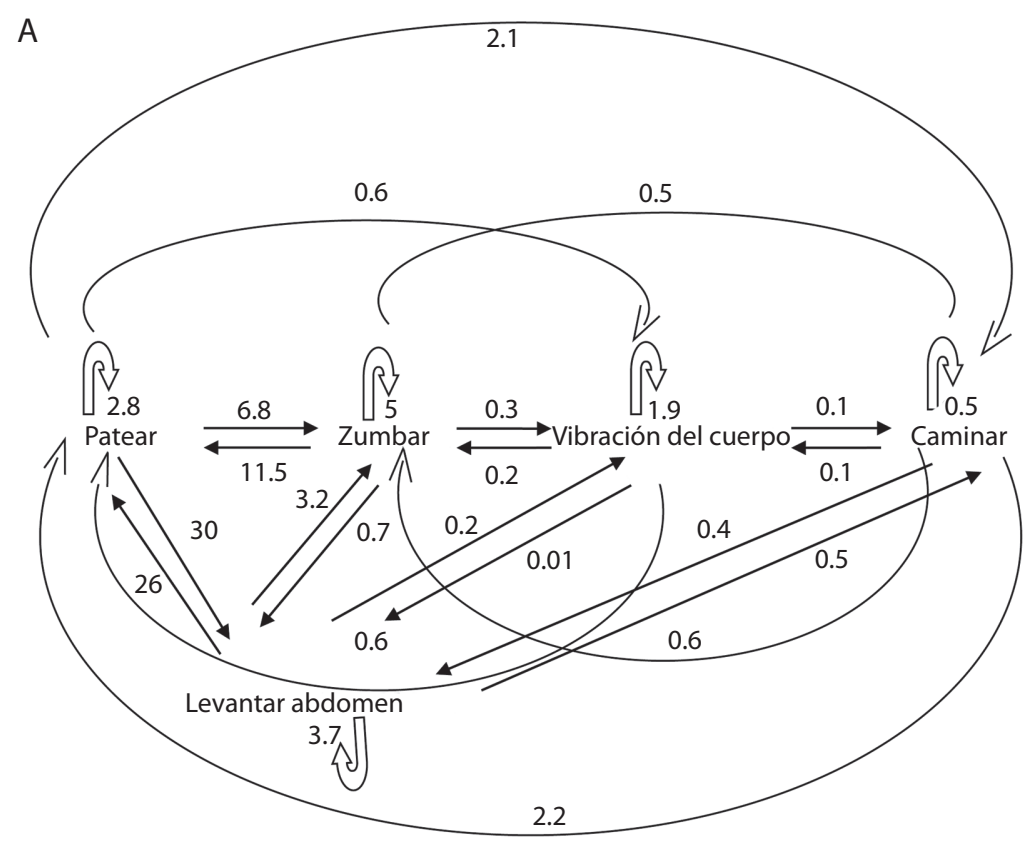

B

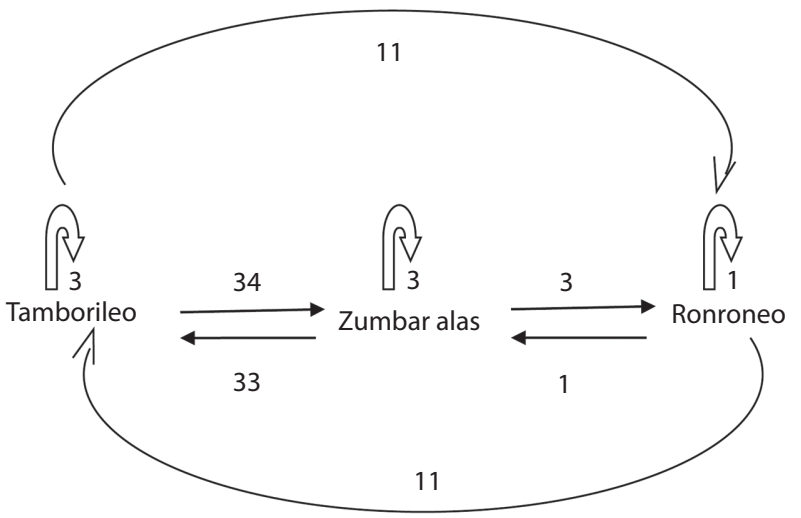

Fig. 2. Las transiciones más frecuentes entre a) comportamientos (6892 actos en 20 cópulas) y b) sonidos durante la cópula en E. floridianus. Los números se refieren a porcentajes de todas las transiciones. Línea curva indica repetición.

Fig. 2. The most frequent transitions between a) behaviors (6892 events in 20 copulations) and b) sounds during mating in E. floridianus. The numbers refer to percentages of all transitions. Curved line indicates repetition.

Ronroneo: Durante el cortejo el ronroneo representó solamente un 3\% del total de los sonidos registrados y se detectó por igual cuando el macho estuvo sobre $(50 \%)$ o cerca de la hembra (50\%). En la cópula, cada estallido de ronroneos duró en promedio $5.8 \pm 1.8 \mathrm{~s}$ con una frecuencia de $131.6 \pm 110.6 \mathrm{~Hz}$ y $19.6 \pm 6.4$ pulsos por ronroneo. La duración promedio de cada pulso fue de $0.5 \pm 1.5 \mathrm{~s}$ y una tasa de $3.6 \pm 1.7$ pulsos por segundo ( 14 cópulas, $\mathrm{n}=42$ ).

Durante la cópula, el ronroneo fue mucho más frecuente y representó el $22 \%$ de los sonidos y se exhibió cuando ambos se encontraban con sus abdómenes levantados, y no así cuando 


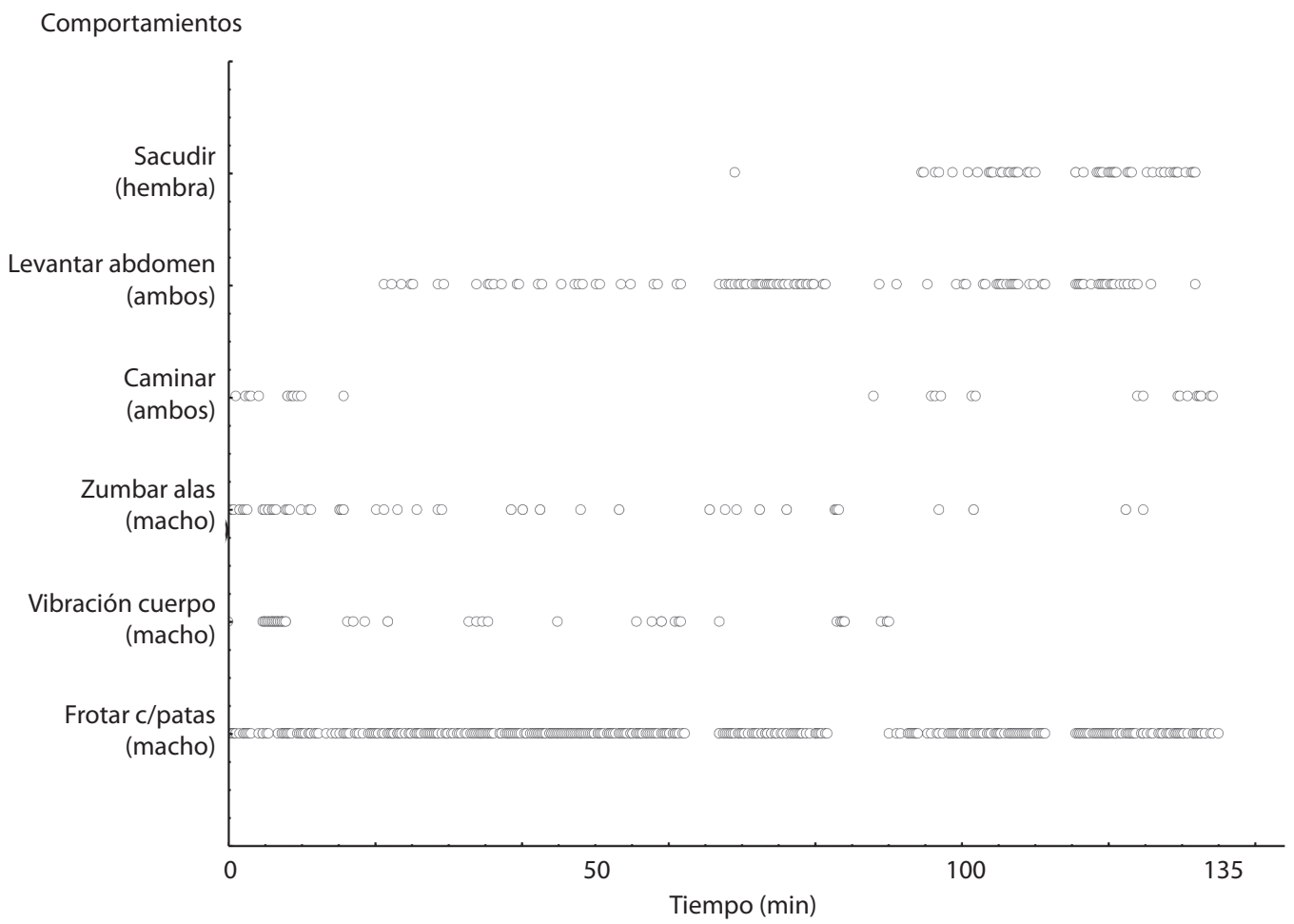

Fig. 3. Comportamientos observados para machos y hembras de E. floridianus durante una cópula, duración 135 minutos. Fig. 3. Behaviors observed for males and females of E. floridianus during copulation, duration 135 minutes.

el macho frotó con sus patas el abdomen de la hembra. Se encontraron diferencias durante el cortejo y la cópula en cuanto a la frecuencia de la señal (hz), número de pulsos y frecuencia (\#pulsos/tiempo) (Cuadro 2). Fue frecuente que el ronroneo fuera seguido del tamborileo y viceversa (Fig. 2b). No se encontró ninguna asociación entre la frecuencia de los ronroneos y agitar o no el cuerpo por parte de la hembra.

Tamborileo: El tamborileo representó el $47 \%$ de los sonidos registrados durante el cortejo, fue frecuente registrarlo cuando el macho se encontraba sobre la hembra $(60 \%)$ pero también cerca de ella (13\%) y aún lejos de ella $(26,5 \%)$. La duración promedio de cada estallido de tamborileo fue de $3.9 \pm 4.1 \mathrm{~s}$ con una frecuencia promedio de $187.6 \pm 85.7 \mathrm{~Hz}$ y $3.9 \pm 4.5$ pulsos por tamborileo. La duración promedio de cada pulso fue de $0.4 \pm 0.7 \mathrm{~s}$ y una tasa de $3.9 \pm 1.9$ pulsos por segundo ( 14 cópulas, $\mathrm{n}=90$ ).
Durante la cópula el tamborileo también se detectó con frecuencia y representó el $49 \%$ de los sonidos registrados, del total un $73 \%$ se registró durante el frote de patas por parte del macho y el restante cuando ambos permanecían con sus abdómenes levantados (13\%). También en este caso el tamborileo exhibido en la cópula, se diferenció significativamente del producido durante el cortejo, en cuanto a la frecuencia de la señal $(\mathrm{Hz})$, duración y el número de pulsos (Cuadro 2), no así en cuanto a la frecuencia (\#pulsos/tiempo). Por otro lado, los tamborileos se volvieron más frecuentes después de que la hembra agitó el abdomen $\left(\mathrm{x}^{2}=11.7, \mathrm{p}<0.001, \mathrm{n}=21\right)$.

\section{Zumbido de alas hacia delante (sonido} audible): La duración promedio de cada zumbido de alas fue de $1.5 \pm 3.7 \mathrm{~s}$ y una frecuencia promedio en la señal de $148.7 \pm 120 \mathrm{~Hz}(14$ cópulas, $\mathrm{n}=73$ ). Durante el cortejo representó 


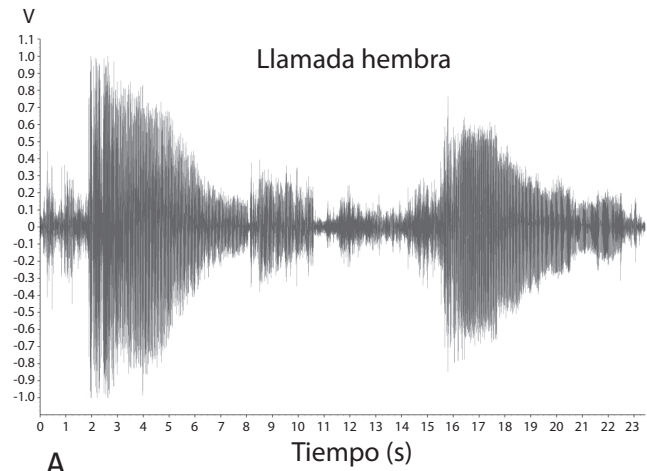

A

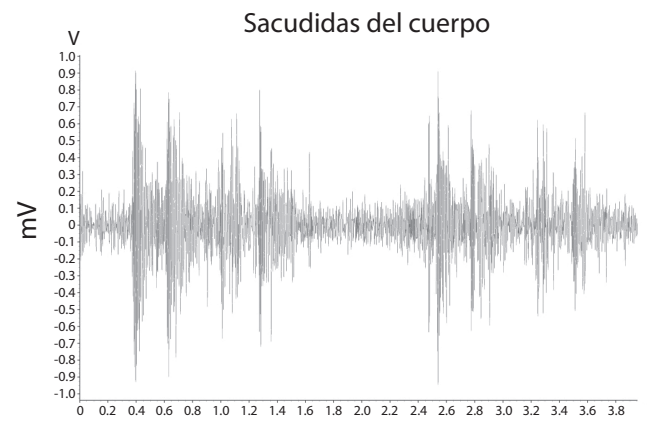

Tiempo (s)

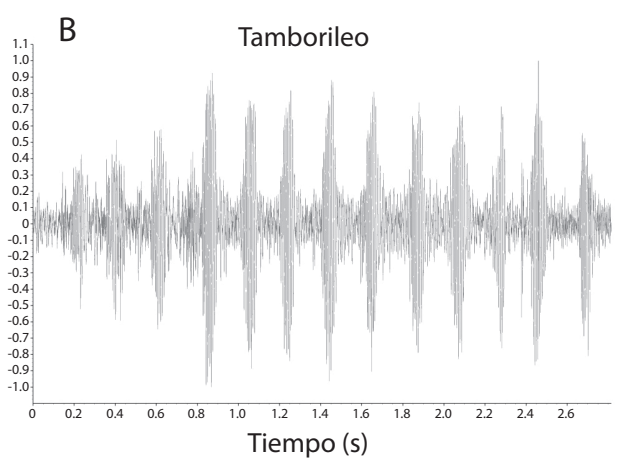

Ronroneo

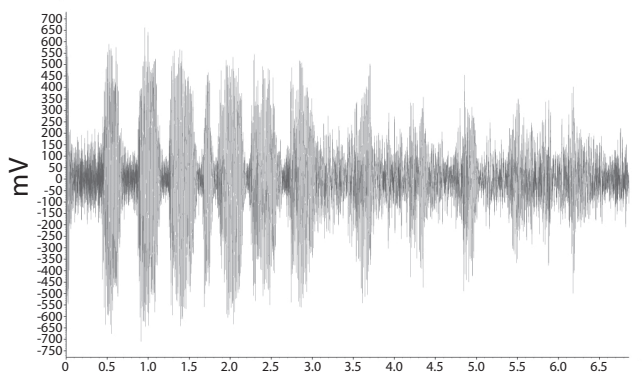

D

Tiempo (s)

Fig. 4. Oscilogramas de las señales vibratorias para: a. Llamada de la hembra, b. Tamborileo, c. Sacudidas rápidas y d. Ronroneo.

Fig. 4. Oscillograms of vibrational signals for: a. Call of the female, b. Drumming, c. Quick jerks of the body and d. Purr.

CUADRO 2

Parámetros de los sonidos (promedio y desviación estándar) producidos durante la cópula y cortejo por los machos de E. floridianus. Prueba de U-Mann Whitney.

TABLE 2

Parameters of the sounds (average and standard deviation) produced during copulation a nd courtship by males of E. floridianus. U-Mann Whitney Test.

\begin{tabular}{lcccccc} 
& \multicolumn{2}{c}{ Ronroneo } & \multicolumn{2}{c}{ Tamborileo } & \multicolumn{2}{c}{ Aleteo } \\
& Cópula & Cortejo & Cópula & Cortejo & Cópula & Cortejo \\
Frec.(Hz) & $131 \pm 110$ & $72 \pm 5^{*}$ & $187 \pm 85$ & $131 \pm 38^{* *}$ & $156 \pm 124$ & $131 \pm 7$ \\
Duración (s) & $5.8 \pm 1.8$ & $5.3 \pm 1.1$ & $3.9 \pm 4.1$ & $14.4 \pm 12^{* *}$ & $1.2 \pm 3.7$ & $8.7 \pm 12$ \\
No. Pulsos & $19.6 \pm 6.4$ & $12.1 \pm 3.2^{* *}$ & $13.9 \pm 14.5$ & $41.6 \pm 28.7^{* *}$ & & \\
& & & & & & \\
No. pulsos/s & $3.5 \pm 1.7$ & $2.3 \pm 0.4^{* *}$ & $3.9 \pm 1.9$ & $3.7 \pm 1.9$ & \\
\hline
\end{tabular}

$*=$ significativa, $\mathrm{p}<0.05$.

$* *=$ altamente significativa, $\mathrm{p}<0.001$. 


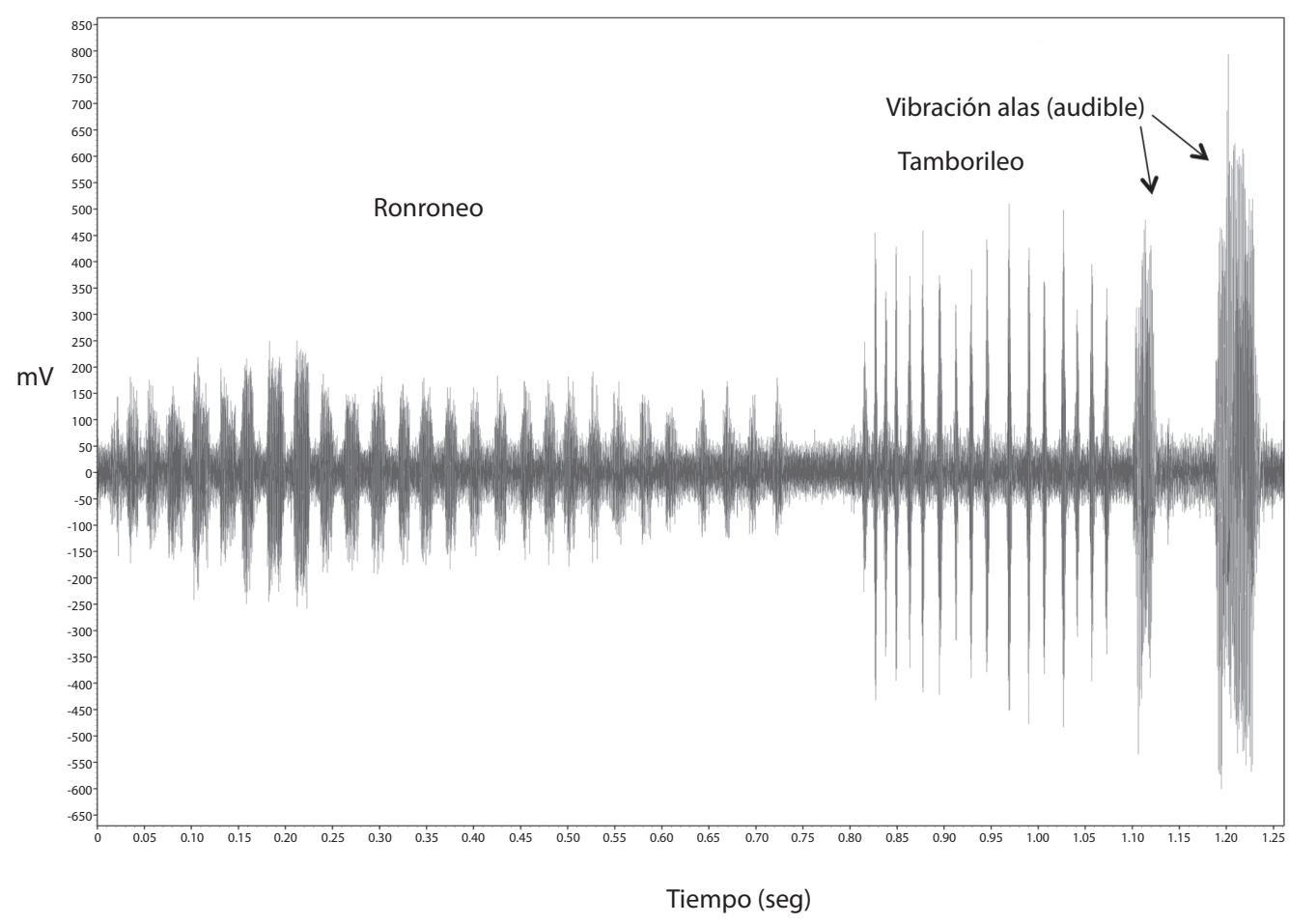

Fig. 5. Oscilograma de la secuencia del canto de un macho durante la cópula.

Fig. 5. Oscillogram of a male song sequence during mating.

el 3\% de los sonidos y ocurrieron cuando el macho se encontraba cerca $(50 \%)$ o sobre la hembra (50\%).

El zumbido de alas representó un 29\% del total de los sonidos durante la cópula y el $89 \%$ de las veces se observó cuando el macho frotaba con sus patas el abdomen de la hembra, y el restante cuando ambos permanecían con el abdomen levantado. No se diferenciaron significativamente las características del sonido (frecuencia y duración) de los zumbidos producidos durante el cortejo y la copula (Cuadro 1). El número de los zumbidos $(72 \pm 39 \mathrm{vs} 25 \pm 18$, $\mathrm{n}=15, \mathrm{p}<0.01)$ y $\mathrm{su}$ frecuencia $(2.4 \pm 1.3 \mathrm{vs}$ $0.8 \pm 0.7, \mathrm{n}=15, \mathrm{p}<0.001)$ tendió a disminuir significativamente conforme avanzó la cópula (Test de Kolgomorov Smirnov).
Experimentos con sonidos emitidos por las hembras: La mayoría de los machos respondieron con el comportamiento de búsqueda a la estimulación con la emisión de la canción de llamada natural de las hembras $(n=18)$. El tiempo de respuesta entre la emisión de la señal y el inicio de la búsqueda fue de $110 \pm 20 \mathrm{~s}$. La mayoría de los machos $(75 \%, \mathrm{n}=13)$ que inició la búsqueda encontró la fuente en un tiempo promedio de $97 \pm 18$ s. Cuando llegaron a la unión entre el tallo principal y los peciolos usualmente colocaron las patas en diferentes tallos. Después de varios segundos, durante los cuales se percibió otra señal de la hembra, siguieron caminando. Los machos emitieron esporádicamente señales vibratorias durante la búsqueda y el $20 \%$ de las señales fueron 
emitidas mientras que los machos estaban caminando. Cuando los machos llegaron a la fuente de vibración, continuaron buscando en la hoja o en el excitador de vibración.

Señales producidas por las hembras. Pitoreta o llamada: Solamente se observó previo al encuentro de los dos sexos y lo produjeron únicamente las hembras. En acercamientos con la cámara de video se determinó que siempre durante el sonido, la hembra levantó sus hemiélitros y los separó ligeramente del abdomen haciéndolos vibrar (Fig. 4). La duración promedio de cada llamada fue de $7.1 \pm 2.4 \mathrm{~s}$ con una frecuencia promedio de $127.3 \pm 84.6 \mathrm{~Hz}$. La frecuencia fue de 31.1 llamadas por segundo con un intervalo promedio entre llamadas de $16.6 \pm 8.9 \mathrm{~s}(\mathrm{n}=23)$.

\section{DISCUSIÓN}

Los patrones temporales y espectrales de señales vibratorias de E. floridianus durante el cortejo fueron similares a las características descritas anteriormente para las especies de Pentatominae. Sin embargo, se encontraron algunas diferencias en la emisión de señales y en el repertorio de canciones. En la mayoría de las especies de pentatómidos, se han descrito dos o tres canciones para machos y hembras (Čokl, Virant-Doberlet \& Stritih, 2000; Čokl \& Vibrant, 2001; Blassioli-Moraes, Laumann, Cokl \& Borges, 2005) con diferentes características temporales y espectrales. En E. floridianus, el repertorio de señales parece ser menos complejo con sólo una señal para las hembra y tres para los machos, en el que no se detectaron duetos durante el cortejo como los descritos para otras especies en Hemiptera (McBrien, Čokl\& Millar, 2002). En la mayoría de las especies hasta ahora estudiadas en Pentatominae, la comunicación vibratoria comienza por canciones de llamada de las hembras que desencadenan en los machos el moverse hacia ellas y cantar. Como se describió anteriormente en otros estudios (Čokl, Virant-Doberlet \& McDowell, 1999; Ota \& Čokl, 1991) los machos se detuvieron regularmente durante la búsqueda y aunque necesitaron en promedio de unos dos minutos para iniciar la búsqueda, la mayoría tuvo éxito en localizar la fuente de la canción de las hembras. Este estudio confirma, al igual que lo observado en otras especies (Čokl et al., 1999; Ota \& Čokl, 1991), de que las señales vibratorias de las hembras tienen efectos atractivos sobre los machos de E. floridianus.

Por primera vez se documenta la producción de señales vibratorias a lo largo de toda la cópula en esta especie, ya que la mayoría de los estudios bioacústicos en Hemiptera se han circunscrito a la producción de sonidos previos a la cópula. Las comparaciones entre las características de las canciones de Euschistus servus y Nezara virula han demostrado diferencias entre especies en los sonidos y se ha sugerido como parte de los mecanismos de comunicación entre individuos de la misma especie (Ota \& Čokl, 1991; Lampson, Young, Jeremy, Ahmad, Richard \& Everett, 2010). Las características promedios del canto estudiadas en E. floridianus en cuanto a la frecuencia dominante, el tiempo de repetición y la forma de la onda son diferentes de las otras especies que se han estudiado, por ejemplo en Euschistus conspersus (McBrien \& Millar, 2003), Euschistus heros (Blassioli-Moraes et al., 2005), Euschistus servus (Lampson et al., 2010) y N. viridula (Čokl et al., 2000). Las diferencias observadas entre especies podrían explicarse en función del reconocimiento de especies. Sin embargo, el hecho de que la producción de sonidos por parte de los machos continúa durante la cópula nos sugiere que probablemente jueguen también un rol de comunicación en el contexto de la selección sexual.

La producción de sonidos en esta especie se asoció principalmente con comportamientos estimulatorios - mecánicos durante el cortejo como el sacudir el cuerpo cerca o sobre la hembra, durante la cópula como el toqueteo de antenas y el frote de patas sobre los lados del abdomen de la hembra o cuando ambos permanecían con el abdomen levantado durante la cópula. Las sonidos en E. floridianus fueron significativamente diferentes en la frecuencia, 
duración y número de pulsos tanto en el cortejo como en la cópula para los sonidos de ronroneo y tamborileo. Es probable que los patrones de conducta de los machos se ajusten a las demandas de las hembras en ciertos momentos durante el cortejo y la cópula, por ejemplo estos tendieron a producir más ronroneos cuando la hembra agitó el abdomen.

Los comportamientos de cortejo en $E$. floridianus no difieren de los observados en otras especies de pentatómidos y aparentemente siguen una serie características de pasos donde exhiben comportamientos similares. Los principales pasos de comportamiento incluyen: 1) un macho se aproxima caminando a una hembra, 2) toqueteo de antenas por el macho a la hembra, 3) la elevación del abdomen por las hembras receptivas, 4) toqueteo antenas y estimulación con la genitalia parcialmente extendida el abdomen de la hembra y 5) cópula final punta-punta (Fish \& Alcock, 1973; Borges, Jepson \& Howse, 1987; Wang \& Millar, 1997). Sin embargo, no hay estudios en Hemiptera que muestren a las vibraciones sísmicas como parte del cortejo durante la cópula y su asociación con otros comportamientos. De acuerdo con Eberhard (1996) en una revisión de la literatura el comportamiento copulatorio de los machos es muy común que suceda en al menos 32\% de 302 especies de artrópodos. Varios aspectos de la conducta del macho observados en E. floridianus se ajustan a los criterios de "cortejo copulatorio" como los de repetición rítmica a lo largo de una cópula y en diferentes cópulas, además de ser apropiados para estimular a la hembra (Eberhard, 1994). Se cree que el cortejo copulatorio funciona para activar en las hembras ciertas respuestas de comportamiento o fisiológicos (tales como transporte de esperma, ovulación, inhibición de aparearse nuevamente, prevenir la intromisión y eyaculación entre otros) que operan en función de los intereses reproductivos del macho (Eberhard, 1996; Briceño \& Eberhard, 2009; Briceño \& Eberbard, 2009a). Wall \& Langley (1993) propusieron una hipótesis alternativa cuyo fin es el de «reforzar la unión copulativa», pero las observaciones realizadas hasta el momento en E. floridianus no ofrecen ninguna indicación de que la conducta del macho afecte el acoplamiento físico de sus genitales. El frote de los machos con sus patas, como se ha sugerido para la mosca tsetse en Glossina morsitans y G. austeni (Huyton \& Langley, 1982), puede tener como función probable el inhibir los desplazamientos de las hembras.

Los patrones de comportamiento y su asociación con las vibraciones de sustrato sugieren que estas señales pueden ser importantes para los machos durante el cortejo y la copula, y que deben prestárseles atención en cuanto a su función en estudios futuros. Unos posibles experimentos para saber si los comportamientos de los machos y las vibraciones están moldeadas por la elección criptica femenina seria asociar la intensidad del cortejo o las señales emitidas por los machos con diferentes respuestas por parte de la hembra como su aceptación, prevenir la intromisión y eyaculación o finalización forzada de la copula. Es importante también en estudios posteriores incluir un mayor número de especies para entender la evolución de la comunicación vibracional en el contexto de la elección críptica por parte de la hembra.

\section{AGRADECIMIENTOS}

A Andrés Araya por colaborar en el análisis y a Laura Chavarría, Natalia Ramírez y Patricia Ortiz en las grabaciones. A los revisores anónimos por sus valiosos comentarios. A Bernal Burgos por la cría de las chinches. A la Vicerrectoría de Investigación por el apoyo económico.

\section{RESUMEN}

Euthyrhynchus floridanus (Linnaeus) (Hemiptera: Pentatomidae) es una especie neotropical perteneciente a la familia Pentatomidae con más de 4000 especies descritas, y se distribuye desde la Florida hasta Brasil. Este estudio describe el comportamiento sexual y reporta por primera vez la producción de vibraciones de sustrato por machos y hembras durante el comportamiento precopulatorio y la cópula. Se grabó tanto el comportamiento de cortejo y de cópula como las diversas señales producidas utilizando un cartucho fonográfico, conectado a una cámara de video. 
Posteriormente se reprodujo las vibraciones de las hembras en ausencia de estas y se registró las respuestas de los machos. Se distinguieron al menos tres vibraciones de sustrato distintas para los machos y una para las hembras, y se caracterizaron por ser señales de baja frecuencia que van desde los 127 hasta $180 \mathrm{~Hz}$. Las sonidos de los machos en E. floridianus fueron significativamente diferentes en la frecuencia, duración y número de pulsos tanto en el cortejo como en la cópula para los sonidos de ronroneo y tamborileo. La producción de sonidos en esta especie se asoció principalmente con comportamientos estimulatorios- mecánicos durante el cortejo y copula. Patrones de comportamiento y su relación con las vibraciones del sustrato sugiere que estas señales pueden ser importantes para los machos en el contexto de la localización de su compañera y la selección sexual.

Palabras clave: Euthyrhynchus floridanus, Hemiptera, chinches, vibraciones de sustrato, cortejo, apareamiento.

\section{REFERENCIAS}

Arismendi, N., \& Thomas, D. B. (2003). Pentatomidae (Heteroptera) of Honduras: a checklist with description of a new ochlerine genus. Insecta Mundi, 17, 219-225.

Barth, F. G. (2002). A Spider's World: Senses and Behaviour. Berlin: Springer.

Blassioli-Moraes, M. C., Laumann, R. A., Cokl, A., \& Borges, M. (2005). Vibratory signals of four Neotropical stink bug species. Physiological Entomology, 30: 175-188.

Borges, M., Jepson, P. C., \& Howse, P. E. (1987). Longrange mate location and close range courtship behavior of the green stink bug, Nezara viridula and its mediation by sex pheromones. Entomologia Experimentalis et Applicata, 44, 205-212.

Briceño, R. D., \& Eberhard, W. (2009). Experimental modifications imply stimulatory functions for male tsetse fly genitalia, supporting cryptic female choice theory. Journal of Evolutionary Biology, 22, 1516-1525.

Briceño, R. D., \& Eberhard, W. (2009a). Experimental demonstrations of possible cryptic female choice on male tsetse fly male genitalia. Journal of Insect Physiology, 55, 989-996.

Cocroft, R. (2001). Vibrational communication and the ecology of group living herbivorus insects. American Zoologist, 41, 1215-1221.

Cocroft, R. B., \& Rodríguez, R. L. (2005). The behavioral ecology of insect vibrational communication. BioScience, 55, 323-334.

Čok1, A., Virant-Doberlet, M., \& McDowell, A. (1999). Vibrational directionality in the southern green stink bug Nezara viridula (L.) is mediated by female song. Animal Behavior, 58, 1277-1283.

Čokl, A., Virant-Doberlet, M., \& Stritih, N. (2000). The structure and function of songs emitted by southern green stink bugs from Brazil, Florida, Italy and Slovenia. Physiological Entomolology, 25, 196-205.

Čokl, A., \& Vibrant-Dobelert, M. (2001). Communication with substrate-borne signals in small plant dwelling insects. Annals of the Entomological Society of America, 97, 471-79.

De Clercq, P. (2000). Predaceous sting bugs (Pentatomidae: Asopinae). In C. W. Schaefer, \& A. R. Panizzi (Eds.), Heteroptera of economic importance (pp. 737-789). Boca Ratón, FL: CRC Press.

Eberhard, W. G. (1994). Evidence for widespread courtship during copulations in 131species of insects and spiders, and implications for cryptic female choice. Evolution, 48, 711-733.

Eberhard, W. G. (1996). Female control: sexual selection by cryptic female choice. Princeton, NJ: Princeton Univ. Press.

Fish, J., \& Alcock, J. (1973). The behavior of Chlorochroaligata (Say) and Cosmopepla bimaculata (Thomas) (Hemiptera: Pentatomidae). Entomolological News, $84,260-268$.

Haskell, N. A. (1953). The dispersion of surface waves on multilayered media. Bulletin of the Seismological Society of America, 43, 17-34.

Huyton, P. M., \& Langley, P. A. (1982). Copulatory behavior of tsetse flies Glossina morsitans and G. austeni. Physiological Entomology, 5, 243-252.

Kalmring, K., \& Elsner, N. (1985). Acoustic and Vibrational Communication in Insects. Berlin: Verlag-Parey.

Lampson, B., Young, H., Jeremy, G., Ahmad, K., Richard, M., \& Everett, F. (2010). Characterization of substrate-borne vibrational signals of Euschistus servus (Heteroptera: Pentatomidae). American Journal of Agricultural and Biological Sciences, 5, 32-36.

McBrien, H. L., Čokl, A., \& Millar, G. (2002). Comparison of substrate-borne vibrational signals of two congeneric stink bug species, Thyanta pallidovirens and T. custator accerra (Heteroptera: Pentatomidae). Journal of Insect Behavior, 15, 715-738.

McBrien, H. L., \& Millard, J. G. (2003). Substrate-borne vibrational signaling in the consperse sting bug, Euschistus conspersus. The Canadian Entomologist, 135, 555-567.

McPherson, J. E., \& McPherson, R. (2010). Stink bugs of economic importance in America north of Mexico. Florida, USA: CRC Press.

Meed, F. W., \& Richman, D. B. (2000). Florida predatory stink bug (unofficial common name), Euthyrhynchus floridanus (Linnaeus) (Insecta: Hemiptera: 
Pentatomidae). Document EENY157, Florida Cooperative Extension Service, Institute of Food and Agricultural Sciences, University of Florida.

Moore, T. E. (1961). Audiospectrographic analysis of sounds of Hemiptera and Homoptera. Annals of the Entomological Society of America, 54, 349-355.

Ota, D., \& Čokl, A. (1991). Mate location in the southern green stink bug Nezara viridula (Heteroptera: Pentatomidae) mediated through substrate-borne signals on Ivy. Journal of Insect Behavior, 4, 441-447.

Rovner, J. S. (1975). Sound production by nearctic wolf spiders: a substratum-coupled stridulating mechanism. Science, 190, 1309-1310.

Stewart, K. W. (1997). Vibrational communication in insects. American Entomologist, 81-91.
Thorpe, K., \& Harrington, J. (1981). Sound production and courtship behavior in the seed bug Ligycornis diffuses. Annals of the Entomological Society of America, 74, 396-373.

Virant-Doberlet, M., \& Čokl, A. (2004). Vibrational communication in insects, Neotropical Entomology, 33, 121-134.

Wall, R., \& Langley, P. A. (1993). The mating behavior of tsetse flies (Glossina): a review Physiological Entomology, 18, 211-218.

Wang, Q., \& Millar, J. G. (1997). Reproductive behavior of Thyanta pallidovirens (Heteroptera: Pentatomidae). Annals of the Entomological Society of America, 90, 380-388. 
